\title{
A Two-stage Stochastic Programming Model for Optimal Reactive Power Dispatch with High Penetration Level of Wind Generation
}

\author{
Wei Cui ${ }^{\dagger}$, Wei Yan*,Wei-Jen Lee**, Xia Zhao*, Zhouyang Ren* and Cong Wang* \\ Abstract - The increasing of wind power penetration level presents challenges in classical optimal \\ reactive power dispatch (ORPD) which is usually formulated as a deterministic optimization problem. \\ This paper proposes a two-stage stochastic programming model for ORPD by considering the \\ uncertainties of wind speed and load in a specified time interval. To avoid the excessive operation, the \\ schedule of compensators will be determined in the first-stage while accounting for the costs of \\ adjusting the compensators (CACs). Under uncertainty effects, on-load tap changer (OLTC) and \\ generator in the second-stage will compensate the mismatch caused by the first-stage decision. The \\ objective of the proposed model is to minimize the sum of CACs and the expected energy loss. The \\ stochastic behavior is formulated by three-point estimate method (TPEM) to convert the stochastic \\ programming into equivalent deterministic problem. A hybrid Genetic Algorithm-Interior Point \\ Method is utilized to solve this large-scale mixed-integer nonlinear stochastic problem. Two case \\ studies on IEEE 14-bus and IEEE 118-bus system are provided to illustrate the effectiveness of the \\ proposed method.
}

Keywords: Costs of adjusting the compensators, Two-stage stochastic programming, Three-point estimate method, Uncertainty, Wind power

\section{Introduction}

The increasing penetration of intermittent and stochastic wind generation presents great challenges for traditional power system control strategies of voltage regulation. To ensure the secure and economic operation of power system, it is necessary to dispatch the reactive sources based upon their regulating characteristics and constraints.

Generally, the compensator (such as capacitor/reactor banks) and on-load tap changer (OLTC) are viewed as the discrete control devices, while the generator is regarded as continuous control devices. The different regulating characteristics of these two types of control devices require distinct control strategies to manage the outcome of voltage profile in the presence of high penetration of wind generation. Due to the daily operation constraints of discrete control devices, frequent and excessive switching operations should be avoided. In [1-6], the daily schedule of discrete control devices is optimized based on dynamic programming approach while accounting for constraints of maximum allowable daily switching operation number (MADSON). The minimum total energy loss of daily 24snapshot is defined as the objective function to evaluate the control performance [5]. In [6], the total power system

$\dagger$ Corresponding Author: Dept. of Electrical Engineering, Chongqing University, Chongqing, China. (cuiwei0626@gmail.com)

* Dept. of Electrical Engineering, Chongqing University, Chongqing, China

** Dept. of Electrical Engineering, The University of Texas at Arlington, Arlington, TX, USA.

Received: December 11, 2015; Accepted: September 1, 2016 operation cost involving fuel cost of generators and switching cost of discrete control devices are considered. However, dynamic programming approach is a very complex nonlinear optimization due to the spatial-temporal coupling. For the sake of simplicity, time-interval-based approaches [7-9] are developed to decompose the daily load curve into several sequential load levels. The number of load levels satisfies the requirement of MADSON constraints. Therefore, this problem is converted into several single Deterministic ORPD (DORPD) problems that can be solved by traditional optimization methods. Another approach considering costs of adjusting discrete control devices is proposed to avoid excessive operations $[6,10]$. However, in these articles, load uncertainty is not taken into account. The schedule of discrete control devices is always solved under a specified load level, which hardly represents the load fluctuation in the corresponding time interval.

Besides, the integration of large amounts of wind generation into power grid brings more uncertainty in real-time operation, which, to a large extent, increases the probability of frequent operation for discrete control devices. If MADSON is reached early in a day, the discrete control devices will be blocked which could degrade the performance of voltage control due to the lack of control capability [5]. As a result, the schedule of discrete control devices based on DORPD without considering the uncertainty may not be the optimal or even feasible.

To deal with this challenge, the stochastic programming incorporated the randomness into the modeling process is 
an alternative. In [11], Chance-constrained programming is utilized in DORPD to handle load uncertainty and random branch outages, the inequality constraints are satisfied by a predefined probability level. In [12], a two-point estimate method is used to model load uncertainty in multiobjective DORPD problem, and statistic estimation method is utilized for the optimal control variables obtained by the repeating deterministic simulation process. The authors in [13] propose a multi-objective optimal active and reactive power dispatch problem with load and wind generation uncertainties. Economic dispatch is used to obtain the real power output of generators. However, in these articles, the different regulating characteristics of discrete and continuous control devices is not considered and differentiated. The discrete control devices will be scheduled associated with the continuous control devices. As a result, MADSON constraints might be violated in daily 24-snapshot application.

In this paper, a two-stage stochastic programming model for ORPD with high penetration level of wind generation is proposed. The main concept of two-stage stochastic programming is that the decision maker takes some action in the first-stage before the actual realization of the uncertainty. Once the uncertainty has unfolded, a recourse decision can be made at the second-stage to coordinate or compensate any negative impact that might have been experienced as a result of the first-stage decision [14-16]. This model is suitable for the coordination of the two different regulating characteristics of control devices. The schedule of discrete control device is preferred to be determined in advance due to the constraints of MADSON, while the continuous control device will cover the uncertainty by continuous adjustment. Since the step size of the OLTC is relatively small, it will be treated as continuous control device in this paper. Specifically, the compensators involving capacitor/reactor banks are regarded as the first-stage integer variables since they are the only reactive source in substation. The OLTC and generator are considered as the second-stage continuous variables which compensate the mismatch caused by the first-stage decision. In other words, such arrangements are to guarantee that there are available resources in either substation or power plant to take care the uncertainty in the real time operation. To model wind speed and load uncertainties in a specified time interval, three-point estimate method (TPEM) is utilized since it only requires the first few statistical moments of the data. The behavior of $m$ random variables can be evaluated by $2 m+1$ deterministic calculation.

From the mathematical point of view, the proposed method is a large-scale mixed-integer nonlinear stochastic programming, which is complex and difficult to solve. In this paper, a hybrid Genetic Algorithm-Interior Point Method [17] is implemented to reduce computational burden caused by stochastic programming. Genetic algorithm (GA), a global optimization technique, is a typical artificial intelligent algorithm with peculiarity of dealing with the integer variables, while interior point method (IPM) achieves a fast convergence to solve largescale nonlinear program problem with a difficult in dealing with discrete variables. The hybrid approach is adopted here to take advantages of both approaches; GA performs the search of switching compensators in the first-stage, while IPM takes control of the continuous variables and constraints in the second-stage under uncertainty formulated by TPEM.

The remaining part of this paper is organized as follows: the proposed two-stage stochastic programming model is introduced in Section III. Then, the stochastic framework based on TPEM is described in Section III. According to the hybrid GA-IPM, the procedure of proposed two-stage stochastic programming is presented in Section 4. Section 5 shows the case study results on the IEEE 14-bus and IEEE 118-bus system. Finally, Section 6 contains a brief conclusion based on the numerical simulation results.

\section{Two-Stage Stochastic Programming Formulation}

In reality, the load pattern and wind farm outputs differed from the forecast always exists, which can pose challenges on classical DORPD. Due to the different regulating characteristics and MADSON constraints, the schedule of discrete and continuous control devices could be determined and deployed in different stages under uncertainty effects. The compensators are classified as the first-stage variables that should be decided before the realization of uncertainty, OLTC and generator are the second-stage variables, which can be decided after the uncertainty have been observed and identified. Therefore, operation times of compensators can be saved due to no response for the uncertainty. This framework is a typical two-stage stochastic programming.

A standard formulation of the two-stage stochastic programming [18] is described as follows:

$$
\begin{gathered}
\min \quad c^{T} x+E[Q(x, \xi)] \quad \text { s.t. } \quad A x=b, x \geq 0 \\
Q(x, \xi)=\min \{q y(\xi) \mid W y(\xi)=h-T x, y(\xi) \geq 0\}
\end{gathered}
$$

where $x$ is known as the first-stage decision variables that should be decided "here-and-now", and it does not response to the uncertainty of $\xi$. On the other hand, $y(\xi)$ denote the second-stage decision variables that are "waitand-see" variables which can be decided when all uncertainty $\xi$ have been observed. Any or all elements of uncertain parameters $\xi=(q, h, T, W)$ can be random. For a given realization of $\xi$, the uncertain parameters in the second-stage become known and the optimal solution $Q(x, \xi)$ shown in Eq. (2) could be carried out consequently. 
The expected value $E[Q(x, \xi)]$ is used to evaluate the recourse cost associated with all realization of $\xi$. The first-stage cost $c^{T} x$ and the recourse cost in the secondstage are involved in the objective function of Eq. (1). To coordinate or compensate with the first-stage decision, the optimal solution tends to have the property that $x$ leaves the second-stage decision in a position to exploit advantageous outcomes of $\xi$.

In general, frequent switching operation could shorten life expectancy of devices and require more maintenance. In this regards, the costs of switching compensators is considered in the first-stage, which aims to avoid excessive operations from the perspective of economics. In the second-stage, the expected energy loss is used to evaluate the control performance of first- and second-stage decision.

Here, the cost of adjusting the compensators (CACs) is represented by unit adjustment cost (UAC), which depends on the investment, the system loss due to the fault of reactive power control devices, etc.[10]. Operating times of compensators is defined as the difference of the bank number switched in. For example, if the current switched capacitor is 2 banks, and the optimal value is 4 , it means two more banks are needed to switch in and the operating time of this capacitor is 2 . Therefore, the cost of switching compensators can be calculated with the UAC multiplied by the corresponding operating times. Suppose that the costs of the recourse decision associated with the adjusting generator terminal voltages and switching tap ratios is neglected.

\subsection{Objective function}

The objective of the optimization is to minimize the sum of costs of switching compensators for the first-stage decision and the expected energy loss through the combined first- and second-stage decisions. The objective function of the two-stage stochastic programming is established as follows:

$$
\begin{gathered}
\min f=\boldsymbol{\alpha}^{T}\left|\boldsymbol{Q}_{\mathrm{C}}-\boldsymbol{Q}_{\mathrm{C}}^{0}\right|+\tau \beta E\left[P_{\text {loss }}\left(\boldsymbol{Q}_{\mathrm{C}}, \boldsymbol{U}, \boldsymbol{\omega}\right)\right] \\
\boldsymbol{U}=\left[\boldsymbol{K}, \boldsymbol{V}_{\mathrm{G}}\right] \\
\boldsymbol{\omega}=\left[\boldsymbol{P}_{\mathrm{L}}, \boldsymbol{Q}_{\mathrm{L}}, \boldsymbol{P}_{\mathrm{W}}, \boldsymbol{Q}_{\mathrm{W}}\right]
\end{gathered}
$$

subject to

$$
\begin{aligned}
& \boldsymbol{g}\left(\boldsymbol{Q}_{\mathrm{C}}, \boldsymbol{U}, \boldsymbol{\omega}\right)=0 \\
& \boldsymbol{Q}_{\mathrm{C}}^{\min } \leq \boldsymbol{Q}_{\mathrm{C}} \leq \boldsymbol{Q}_{\mathrm{C}}^{\max } \\
& \mathbf{V}_{\mathrm{G}}^{\min } \leq \mathbf{V}_{\mathrm{G}} \leq \mathbf{V}_{\mathrm{G}}^{\max } \\
& \boldsymbol{K}^{\min } \leq \boldsymbol{K} \leq \boldsymbol{K}^{\max } \\
& \boldsymbol{Q}_{\mathrm{G}}^{\min } \leq \boldsymbol{Q}_{\mathrm{G}} \leq \boldsymbol{Q}_{\mathrm{G}}^{\max } \\
& \boldsymbol{V}^{\min } \leq \boldsymbol{V} \leq \boldsymbol{V}^{\max }
\end{aligned}
$$

with the following nomenclature:
$\boldsymbol{Q}_{\mathrm{C}}$ vector of numbers of switching compensators as the first-stage decisions;

$\boldsymbol{Q}_{\mathrm{C}}^{0} \quad$ vector of initial numbers of switching compensators;

$\boldsymbol{U}$ vector of second-stage decisions;

$\omega$ vector of uncertain parameters;

$\boldsymbol{P}_{\mathrm{L}} \quad$ vector of real load demands (MW);

$\boldsymbol{Q}_{\mathrm{L}} \quad$ vector of reactive load demands (MVar);

$\boldsymbol{P}_{\mathrm{w}}$ vector of real power output for wind farms (MW);

$\boldsymbol{Q}_{\mathrm{w}}$ vector of reactive power output for wind farms (MVar);

$\boldsymbol{Q}_{\mathrm{G}}$ vector of reactive power output for generators (MVar);

$\boldsymbol{K}$ vector of transformer tap ratios;

$V_{\mathrm{G}}$ vector of generator terminal voltages;

$V$ vector of load bus voltage magnitudes;

$\alpha$ unit adjustment cost of switching compensators (\$/times);

$P_{\text {loss }}$ real power loss (MW);

$\beta$ electricity price $(\$ / \mathrm{kWh})$;

$\tau$ time interval, usual $1 \mathrm{~h}$.

Subscript min and max are the lower and upper bounds, respectively; $E($.$) and \boldsymbol{g}($.) are the expected value operator and load flow function, respectively; In this objective function (3), the first term is regarded as the first-stage function which represents the costs of adjusting the compensators while considering the constraint (7). The second term is regarded as the second-stage function which represents the expected energy loss in the time interval $\tau \tau$, while considering the constraints (6), (8)-(11). $P_{\text {loss }}$ is obtained by calculating the difference between total real power output of generators and total real load demand. Note that the second-stage function is a typical DORPD problem. The corresponding second-stage decisions $\boldsymbol{U}$ will be carried out after the first-stage decisions and uncertain parameters $\omega$ are realized. For a given $\boldsymbol{Q}_{\mathrm{C}}, \boldsymbol{U}$ and $\omega$, state variables involving the voltage magnitude, angle of all load buses and $\boldsymbol{Q}_{\mathrm{G}}$ can be calculated with Eq. (6).

As a classic reactive power dispatch problem, real power economic dispatch is not addressed in this paper. However, it's unreasonable to use one swing bus to balance the system power mismatch caused by the uncertainty. Therefore, real power output of all generators is rescheduled based on the unit participation factors, which is proportional to the original total real power outputs of all generators. Besides, the total real power output of all generators is related with the total load demand, and will be changed proportionally with the fluctuated total load demand. For the wind farm, it is treated as a load with negative real power injection, and not required to match the load demand.

In application, the first-stage decision for the compensators could be implemented and fixed in the time intervals. It means there is no need for compensators to cover uncertainty effects. However, the second-stage 
decision will be determined in real time in which the uncertainty is identified.

\subsection{Normalization of unit adjustment cost}

To demonstrate the objective function with unit power, the normalization is achieved by

$$
\boldsymbol{c}^{T}=\frac{\boldsymbol{\alpha}^{T}}{\tau \beta}
$$

where $c$ denotes the vector of unit adjustment costs of switching compensators based on power (kW/times). Although the UAC of compensators can be evaluated theoretically, it is difficult and unpractical to determine the exact value for each compensator. Therefore, the average value of UAC for all compensators is adopted here. According to long-term operation experience, the average value corresponds to $4 \mathrm{~kW} /$ times [10].

Then, the objective function (3) can be transformed as follows:

$$
\min \quad F=\frac{f}{\tau \beta}=\boldsymbol{c}^{T}\left|\boldsymbol{Q}_{\mathrm{C}}-\boldsymbol{Q}_{\mathrm{C}}^{0}\right|+E\left[P_{\mathrm{loss}}\left(\boldsymbol{Q}_{\mathrm{C}}, \boldsymbol{U}, \boldsymbol{\omega}\right)\right]
$$

\subsection{Wind power model}

Wind power output is related with wind speed. If the wind speed is lower than the cut-in or higher than the cutout wind speed, wind farm will not generate power. At a specified wind speed, the wind farm output $P_{\mathrm{W}}$ can be described as

$$
P_{\mathrm{w}}= \begin{cases}0, & \text { if } 0 \leq v \leq v_{\mathrm{ci}} \\ k_{1} v+k_{2}, & \text { if } v_{\mathrm{ci}}<v \leq v_{\mathrm{r}} \\ P_{\mathrm{r}}, & \text { if } v_{\mathrm{r}}<v \leq v_{\mathrm{co}} \\ 0, & \text { if } v>v_{\mathrm{co}}\end{cases}
$$

where,

$$
\begin{aligned}
& k_{1}=\frac{P_{\mathrm{r}}}{V_{\mathrm{r}}-V_{\mathrm{ci}}} \\
& k_{2}=-k_{1} V_{\mathrm{ci}}
\end{aligned}
$$

where $P_{\mathrm{w}}$ is the wind farm output, $v$ is the average wind speed of wind farm; $P_{\mathrm{r}}$ is the rated capacity of the wind farm; $V_{\text {ci }}, V_{\mathrm{r}}$ and $V_{\text {co }}$ are the cut-in, rated and cut-out speeds of the wind farm, respectively.

Doubly Fed Induction Generators (DFIGs) are widely used in wind farm and have certain reactive compensation capabilities. For simplicity, PQ bus is adopted to represent the characteristic of this kind of wind turbine. Suppose that the power factor of wind turbine is constant with the support of other reactive power source, i.e. capacitor banks, Static Var Compensator, or Static Synchronous Compensator. The reactive power of wind farm is defined as

$$
Q_{\mathrm{w}}=P_{\mathrm{w}} \tan \alpha
$$

where $\alpha$ is power factor angle and $Q_{\mathrm{w}}$ is the reactive power of the wind farm.

\section{Uncertainty Characterization}

The techniques of solving stochastic programming can be classified into three categories: (1) Monte Carlo Simulation (MCS), (2) analytical techniques, and (3) approximate techniques. MCS uses randomized numerical experiments to obtain the distributions of possible outcomes without simplification. However, it requires a large number of iteration to reach the desired convergence [19]. To overcome the deficiency of MCS, analytical techniques use approximate formulas for calculating the statistical moments of the output variables. However, the specific simplification is based on the used formulation which needs significant efforts. Point estimate method (PEM) is the most efficient approximate technique overcomes the limitation mentioned before. To reduce the computational burdens and take advantages the merit of MCS, only certain special points determined by the first few statistical moments are used in PEM. In this paper, the three-point estimate (TPEM) method is utilized here to capture the uncertainty effects [20].

Mathematically, the second-stage function in Eq. (13) is a typical DORPD problem which can be formulated as a nonlinear mapping as follows:

$$
S=s(\boldsymbol{x})
$$

where $\boldsymbol{x}$ is the input random vector includes wind speed and load uncertainties, $S$ is the output vector of the DORPD problem. The task of TPEM is to calculate the raw moments of each output with three points on each of the probability distributions of wind speed and load [20]. Suppose that there are $m$ uncorrelated input variables. For each input variable $x_{i}(i=1,2, \ldots m)$ can be defined as three representative points,

$$
x_{i k}=\mu_{i}+\xi_{i k} \delta_{i} \quad(k=1,2,3)
$$

where $\mu_{i}$ and $\delta_{i}$ are the mean and standard deviation of $x_{i}$. $\xi_{i k}$ is the coefficient reflecting the $k$ th location on its probability distribution.

In Eq. (19), the three values of $\xi_{i k}$ is calculated by

$$
\xi_{i 1}, \xi_{i 2}=\frac{\lambda_{i 3}}{2} \pm \sqrt{\lambda_{i 4}-\frac{3}{4} \lambda_{i 3}^{2}}, \quad \xi_{i 3}=0
$$


where $\lambda_{i 3}$ and $\lambda_{i 4}$ denote the skewness and kurtosis of the random input variable $x_{i}$, respectively, which are calculated by

$$
\begin{aligned}
& \lambda_{i 3}=\frac{\int_{-\infty}^{+\infty}\left(x_{i}-\mu_{i}\right)^{3} f_{i} d x_{i}}{\delta_{i}^{3}} \\
& \lambda_{i 4}=\frac{\int_{-\infty}^{+\infty}\left(x_{i}-\mu_{i}\right)^{4} f_{i} d x_{i}}{\delta_{i}^{4}}
\end{aligned}
$$

where $f_{i}$ is the probability density function of $x_{i}$.

Once the three values of $\xi_{i k}$ for each random input variable $x_{i}$ are obtained, the typical DORPD problem can be calculated three times for each $x_{i}$, while other random variables are specified as their means. Note that only $2 m+1$ times DORPD calculation is performed since $\xi_{i 3}$ is equal to zero. Therefore, this approach converts the stochastic programming with $m$ random input variables into $2 m+1$ equivalent deterministic optimizations.

The raw moment of a random output variable $S$ is estimated by

$$
r_{s}=E[S]=\sum_{i=1}^{m} \sum_{k=1}^{3} w_{i k} S_{i k}
$$

where $w_{i k}$ is three weighting factors calculated by

$$
\begin{aligned}
w_{i 1} & =\frac{1}{\xi_{i 1}^{2}-\xi_{i 1} \xi_{i 2}} \\
w_{i 2} & =\frac{1}{\xi_{i 2}^{2}-\xi_{i 1} \xi_{i 2}} \\
w_{i 3} & =\frac{1}{m}-\frac{1}{\lambda_{i 4}-\lambda_{i 3}^{2}}
\end{aligned}
$$

\section{Mathematical Algorithms}

According to structure of two-stage stochastic programming, a hybrid GA-IPM is developed here. For GA, each individual representing the switching compensators is encoded as the first-stage integer variables and its length is equal to the number of the first-stage variables. For the second-stage function in eq. (13), the expected real power loss can be converted to $2 m+1$ equivalent DORPD. Although IPM is utilized to solve the typical large-scale nonlinear programming problem, $2 m+1$ DORPD running is required for fitness evaluation of each individual. Therefore, the computational burden caused by GA and stochastic programming can be reduced.

An alternative is to take advantage of the intrinsically parallel nature of GA and perform the evaluation of fitness function for all individuals in Master-slave model [21].
Furthermore, A DORPD quadratic model [22] is applied here to speed up calculation. This method replaces OLTC transformer branch with an ideal transformer and its series impedance with dummy node located between them. Therefore, the Hessian matrices become constants and the repeated calculation in iteration process can be avoided.

IPM solves the continuous optimization involving tap ratios and generator terminal voltages under the fixed first-stage variable. In rare cases, the result may be not convergent due to the random first-stage variable. It means the optimal process is infeasible at this operating condition. Therefore, an alternative is to use power flow to calculate the real power loss at this operating condition, which always converges in few iterations.

The basic procedure for proposed two-stage stochastic programming based on the hybrid GA-IPM is summarized as follows:

1) Initial the data including wind speed and load in a specified time interval;

2) Calculate $\lambda_{\mathrm{ik}}, w_{\mathrm{ik}}, \xi_{\mathrm{ik}}$ according to the input data;

3) Create the first generation of switching compensators while considering the constraint (7);

4) Use IPM to calculate the expected real power loss for all individuals in parallel;

5) Evaluate the fitness function based on Eq. (13);

6) Generate a new generation with selection, crossover, mutation operators;

7) Repeat the step 4-6 until user-specified max number of evolutions is reached.

\section{Case Study}

In this section, the IEEE 14-bus and IEEE 118-bus system [23] are used as examples to validate the effectiveness of the proposed method. In both test systems, a one-hour load fluctuation of a real power system is used to simulate the stochastic behavior of the loads. The load demand in every $5 \mathrm{~min}$ is assigned to each load buses according to the initial load proportion of each test system. The curve of one-hour load demand is shown in Fig. 1.

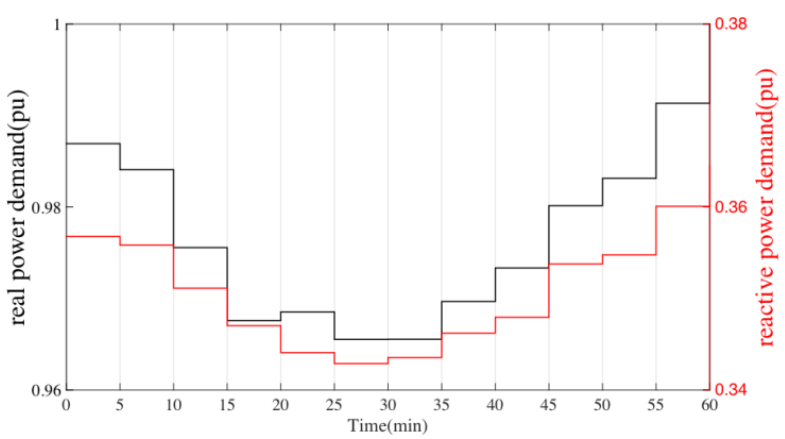

Fig. 1. The load demand in every $5 \mathrm{~min}$ of a real power system. 
Table 1. Location and rated capacity of wind farm

\begin{tabular}{cccc}
\hline Test systems & $\begin{array}{c}\text { Connected } \\
\text { location }\end{array}$ & $\begin{array}{c}\text { Rated } \\
\text { Capacity(MW) }\end{array}$ & $\begin{array}{c}\text { Rated power } \\
\text { factor }\end{array}$ \\
\hline $\begin{array}{c}\text { IEEE 14-Bus } \\
\text { system }\end{array}$ & Bus 8 & 30 & 0.9 \\
$\begin{array}{c}\text { IEEE 118-Bus } \\
\text { system }\end{array}$ & Bus 59 & 260 & 0.9 \\
\hline
\end{tabular}

Table 2 Probability distributions and parameters of wind speed

\begin{tabular}{ccc}
\hline Probability distribution function & $k$ & $c$ \\
\hline$f_{v}(v)=\left(\frac{k}{c}\right)\left(\frac{v}{c}\right)^{k-1} \exp \left[-\left(\frac{v}{c}\right)^{k}\right]$ & 1.637 & 5.218 \\
\hline Cut-in speed(m/s) & Rated speed(m/s) & Cut-out speed(m/s) \\
\hline 3 & 13 & \multicolumn{2}{c}{25} \\
\hline
\end{tabular}

A wind farm compatible to the total capacity of the test system is added. The connected location and the rated capacity of the wind farm are listed in Table 1. It is assumed that the wind speed follows the Weibull distribution which is recognized as the most popular distribution to represent wind speed. The probability distribution function and the parameters of wind speed are illustrated in Table 2.

The modified IEEE 14-bus system includes three generators, one wind farm, four capacitor banks, and three transformers with on-load tap changer. The four capacitor banks are available at bus 3,6,8 and 9, respectively, in which consists of nine 3 MVar steps. The tap settings of transformers are $1.0 \pm 8 \times 1.25 \%$. Bus 1 is assigned as the slack bus within the reactive power controllable range of $[-0.2,0.1]$, bus 2 and 6 are the PV buses within reactive power controllable ranges of $[-0.4,0.5],[-0.2,0.24]$, respectively. The range of the generator voltage is $[0.9,1.1]$.

The modified IEEE 118-bus system consists of fiftythree generators, one wind farm, fourteen compensators and nine transformers with on-load tap changer. Capacitor banks are installed at bus $34,44,45,46,48,74,79,82,83$, 105,107 and 110, and reactor banks are installed at bus 5 and 37. Also, all compensators include nine 5 MVar steps. The transformers have the same parameters as IEEE 14-bus system. Other initial operating conditions and settings of this system are given in [23].

To clearly illustrate the effectiveness of proposed method, a comparison of three different cases has been utilized,

1. DORPD without considering the MADSON constraints;

2. The proposed method considering the uncertainty and CACs;

3. DORPD based on time-interval approach

\subsection{IEEE 14-bus system}

\section{Case 1}

Due to the unlimited operating times, DORPD can make full use of the control capability of discrete control devices. In China, DORPD cycle is executed from $15 \mathrm{~min}$ to $1 \mathrm{~h}$
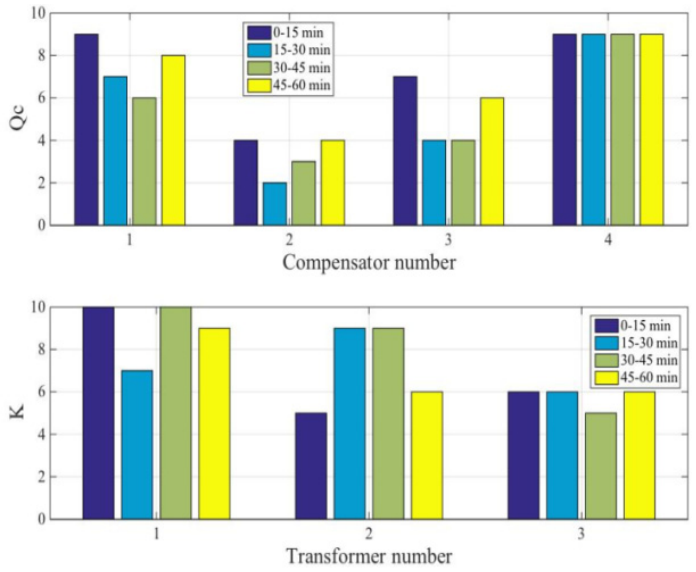

Fig. 2. The schedule of discrete control devices in Case 1

Table 3. Optimization result of Case 1

\begin{tabular}{cccccc}
\hline Time $(\min )$ & $P_{\text {loss }}$ & $Q_{\mathrm{G} 1}$ & $Q_{\mathrm{G} 2}$ & $Q_{\mathrm{G} 6}$ & $\bar{V}_{\mathrm{L}}$ \\
\hline 0 & 0.112393 & -0.0546 & 0.2987 & 0.0364 & 1.0526 \\
5 & 0.111857 & -0.0534 & 0.2959 & 0.0339 & 1.0526 \\
10 & 0.110276 & -0.0496 & 0.2874 & 0.0265 & 1.0526 \\
15 & 0.081897 & 0.0187 & 0.1938 & 0.0220 & 1.0534 \\
20 & 0.082017 & 0.0184 & 0.1945 & 0.0227 & 1.0534 \\
25 & 0.081633 & 0.0194 & 0.1923 & 0.0205 & 1.0534 \\
30 & 0.068949 & 0.0545 & 0.1385 & -0.0741 & 1.0538 \\
35 & 0.069339 & 0.0533 & 0.1408 & -0.0717 & 1.0538 \\
40 & 0.069731 & 0.0521 & 0.1432 & -0.0693 & 1.0538 \\
45 & 0.096364 & -0.0176 & 0.2396 & -0.0129 & 1.0530 \\
50 & 0.096829 & -0.0187 & 0.2421 & -0.0106 & 1.0530 \\
55 & 0.098117 & -0.0220 & 0.2492 & -0.0041 & 1.0530 \\
\hline
\end{tabular}

according to the daily load demand prediction [24]. Theoretically, the shorter execution cycle used, the better voltage profile can be achieved.

In this case, DORPD execution cycle is assumed as 15 min, which means the schedule of discrete control devices will be implemented and fixed in the $15 \mathrm{~min}$ interval. However, generators will be adjusted automatically to satisfy the load demand. The minimization of real power loss is considered as the objective function which ignores the costs of adjusting the discrete control devices. The optimal schedule of discrete control devices in each time interval is shown in Fig. 2, and the control performance is listed in Table 3. The real power loss of each time interval $\mathrm{P}_{\text {loss }}$, generator reactive power outputs $\mathrm{Q}_{\mathrm{G}}$, the mean value of voltage for all load buses $\bar{V}_{\mathrm{L}}$ are investigated here.

From Fig. 2, it is obviously that discrete control devices are switched to the ideal position based on DORPD in each $15 \mathrm{~min}$ interval. The tap ratio is rounded to the nearest integral tap position due to the relatively small step. Note that compensator 4 is fully injected within the hour due to relatively large reactive power demand in this location, however, the schedule of other compensators varies frequently within the present boundaries. Therefore, the MADSON might be exceeded if this scheme is applied into the whole day. It is observed from Table 3 that generators produce adequate reactive powers to take the 
responsibility of balancing reactive power in the case of the fixed schedule of discrete control devices. Also, both $\mathrm{P}_{\text {loss }}$ and $\bar{V}_{\mathrm{L}}$ are regulated well in each DORPD cycle.

\section{Case 2}

The proposed two-stage stochastic programming is to capture the stochastic behavior of the wind speed and load within the hour. The initial numbers of switching compensators could be achieved from Case 1 in $0-15$ interval simulation results. For GA, the population size is 100 , crossover rate is 0.7 , and mutations rate is 0.2 . When the number of generations reaches 100, GA will stop automatically. For IPM, $2 m+1$ times DORPD is used to evaluate the expected real power loss while considering the constraints.

Based on Eq. (13), the first-stage decision for com-
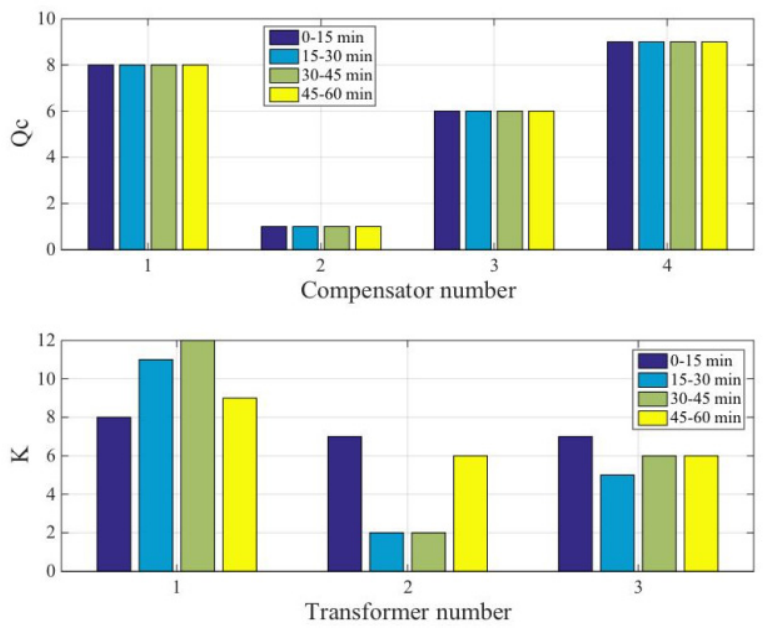

Fig. 3. The schedule of discrete control devices in Case 2

Table 4. Optimization result of Case 2

\begin{tabular}{|c|c|c|c|c|c|}
\hline Time(min) & $P_{\text {loss }}$ & $Q_{\mathrm{G} 1}$ & $Q_{\mathrm{G} 2}$ & $Q_{\mathrm{G} 6}$ & $\bar{V}_{\mathrm{L}}$ \\
\hline 0 & $\begin{array}{c}0.112460 \\
(0.06 \%)\end{array}$ & -0.0569 & 0.3271 & 0.1561 & $\begin{array}{c}1.0529 \\
(0.03 \%)\end{array}$ \\
\hline 5 & $\begin{array}{c}0.111920 \\
(0.06 \%)\end{array}$ & -0.0557 & 0.3242 & 0.1535 & $\begin{array}{c}1.0529 \\
(0.03 \%)\end{array}$ \\
\hline 10 & $\begin{array}{c}0.110329 \\
(0.05 \%)\end{array}$ & -0.0519 & 0.3157 & 0.1459 & $\begin{array}{c}1.0529 \\
(0.03 \%)\end{array}$ \\
\hline 15 & $\begin{array}{c}0.081935 \\
(0.05 \%)\end{array}$ & 0.0206 & 0.1684 & 0.0290 & $\begin{array}{c}1.0520 \\
(-0.14 \%)\end{array}$ \\
\hline 20 & $\begin{array}{c}0.082054 \\
(0.05 \%)\end{array}$ & 0.0203 & 0.1690 & 0.0294 & $\begin{array}{c}1.0520 \\
(-0.14 \%)\end{array}$ \\
\hline 25 & $\begin{array}{c}0.081673 \\
(0.05 \%)\end{array}$ & 0.0213 & 0.1670 & 0.0279 & $\begin{array}{c}1.0518 \\
(-0.15 \%)\end{array}$ \\
\hline 30 & $\begin{array}{c}0.069036 \\
(0.13 \%)\end{array}$ & 0.0579 & 0.0936 & -0.0434 & $\begin{array}{c}1.0511 \\
(-0.26 \%)\end{array}$ \\
\hline 35 & $\begin{array}{c}0.069432 \\
(0.13 \%)\end{array}$ & 0.0568 & 0.0959 & -0.0411 & $\begin{array}{c}1.0511 \\
(-0.26 \%)\end{array}$ \\
\hline 40 & $\begin{array}{c}0.069820 \\
(0.13 \%)\end{array}$ & 0.0556 & 0.0982 & -0.0388 & $\begin{array}{c}1.0511 \\
(-0.26 \%)\end{array}$ \\
\hline 45 & $\begin{array}{c}0.096364 \\
(0.00 \%)\end{array}$ & -0.0176 & 0.2396 & 0.0771 & $\begin{array}{c}1.0530 \\
(0.00 \%)\end{array}$ \\
\hline 50 & $\begin{array}{c}0.096829 \\
(0.00 \%)\end{array}$ & -0.0187 & 0.2421 & 0.0794 & $\begin{array}{c}1.0530 \\
(0.00 \%)\end{array}$ \\
\hline 55 & $\begin{array}{c}0.098117 \\
(0.00 \%)\end{array}$ & -0.0220 & 0.2492 & 0.0859 & $\begin{array}{c}1.0530 \\
(0.00 \%)\end{array}$ \\
\hline
\end{tabular}

pensators is carried out and will be implemented during the hour. After uncertainty is unfolded, the second-stage decision will be obtained from DORPD execution cycle with the same objective function as Case 1. Then, the schedule of discrete control devices will be performed together in the $15 \mathrm{~min}$ interval. Similarly, generators are adjusted according to load demand. The optimization results are presented in Fig. 3 and Table 4.

From the results above, the schedule of compensators is fixed during the hour and compensator 4 has the same schedule with Case 1, which means the proposed method can provide an appropriate schedule for compensator 4 under uncertainty effects. The tap ratios and generator reactive outputs are both far away from the boundaries in each interval. In other words, the changed dispatch of reactive power caused by the fixed schedule of compensators can be balanced and coordinated appropriately.

Compared with Case 1, $P_{\text {loss }}$ and $\bar{V}_{\mathrm{L}}$ in Case 2 are close match in most instances (the difference between two cases is shown in the bracket). The small differences indicate that the proposed method can achieve a similar voltage profile. A relatively large difference of $P_{\text {loss }}$ and $\bar{V}_{\mathrm{L}}$ is observed from Table 4 in 30-45 min interval. Since the load passed into off-peak level in this period, which results in a relatively large difference in compensators schedule between Case 2 and Case 1.

\section{Case 3}

To demonstrate the effects of proposed method, comparison study is performed. In this case, the schedule of compensators is determined based on the idea of timeinterval-based approach. The mean value is used to represent the one-hour load demand. Then, the schedule of compensators as the first-stage decision is carried out by DORPD. The second-stage decision will be obtained from the same procedure mentioned in Case 2.

As shown in Fig. 4, the schedule of compensators is kept at constant during the hour but in different switching numbers compared with Case 2 . In addition, $P_{\text {loss }}$ and $\bar{V}_{\mathrm{L}}$
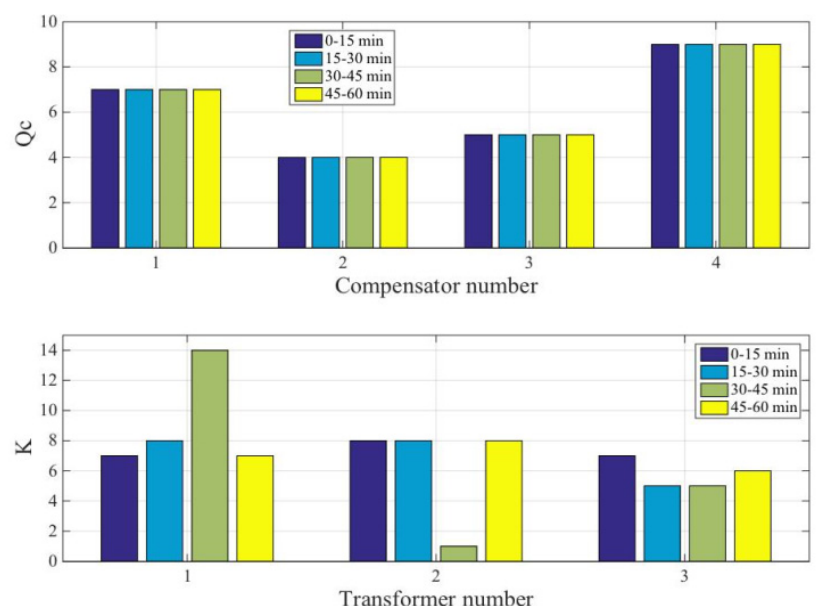

Fig. 4. The schedule of discrete control devices in Case 3 
are increased or reduced to a different extent according to the difference between Case 3 and Case 1 .

For comparison purpose, the results of three cases are summarized together in Table 6. The total operating times of compensators $N_{\mathrm{C}}$ and OLTC $N_{\mathrm{K}}$, the mean value of real power loss $\bar{P}_{\text {loss }}$, the mean value of voltage of all load buses $\bar{V}$ and standard deviation of generator reactive power outputs $D\left(Q_{\mathrm{G}}\right)$ are investigated here.

To calculate $\mathrm{N}_{\mathrm{K}}$, the result of each case in 0-15 interval is supposed as the initial tap ratios of transformers. Operating times of an OLTC is defined as the difference of tap ratios. In Case $2, \mathrm{~N}_{\mathrm{C}}$ is reduced about $64 \%$ from Table 6, which indicates that the proposed method considering CACs can avoid excessive operations of compensators under uncertainty effects. On the other hand, the response of generators (relatively larger $\mathrm{D}\left(\mathrm{Q}_{\mathrm{G}}\right)$ ) and OLTC (acceptably increased $\mathrm{N}_{\mathrm{K}}$ ) show that the secondstage decision can effectively handle the uncertainty. Note that the total operating times of compensators and OLTC is also reduced.

In Case 3, $N_{\mathrm{C}}$ is reduced even more than Case 2 since the inappropriate representation of the one-hour load

Table 5. Optimization result of Case 3

\begin{tabular}{|c|c|c|c|c|c|}
\hline $\begin{array}{l}\text { Time } \\
(\min )\end{array}$ & $P_{\text {loss }}$ & $Q_{\mathrm{G} 1}$ & $Q_{\mathrm{G} 2}$ & $Q_{\mathrm{G} 6}$ & $\bar{V}_{\mathrm{L}}$ \\
\hline 0 & $\begin{array}{c}0.112617 \\
(0.20 \%)\end{array}$ & -0.0594 & 0.3570 & 0.0993 & $\begin{array}{c}1.0526 \\
(0.00 \%)\end{array}$ \\
\hline 5 & $\begin{array}{c}0.112073 \\
(0.19 \%)\end{array}$ & -0.0581 & 0.3540 & 0.0967 & $\begin{array}{c}1.0526 \\
(0.00 \%)\end{array}$ \\
\hline 10 & $\begin{array}{c}0.110471 \\
(0.18 \%)\end{array}$ & -0.0543 & 0.3454 & 0.0889 & $\begin{array}{c}1.0526 \\
(0.00 \%)\end{array}$ \\
\hline 15 & $\begin{array}{c}0.081930 \\
(0.04 \%)\end{array}$ & 0.0188 & 0.1862 & -0.0598 & $\begin{array}{c}1.0530 \\
(-0.04 \%)\end{array}$ \\
\hline 20 & $\begin{array}{c}0.082049 \\
(0.04 \%)\end{array}$ & 0.0185 & 0.1869 & -0.0591 & $\begin{array}{c}1.0530 \\
(-0.04 \%)\end{array}$ \\
\hline 25 & $\begin{array}{c}0.081666 \\
(0.04 \%)\end{array}$ & 0.0196 & 0.1847 & -0.0612 & $\begin{array}{c}1.0530 \\
(-0.04 \%)\end{array}$ \\
\hline 30 & $\begin{array}{c}0.068999 \\
(0.07 \%)\end{array}$ & 0.0562 & 0.1209 & -0.1051 & $\begin{array}{c}1.0487 \\
(-0.49 \%)\end{array}$ \\
\hline 35 & $\begin{array}{c}0.069395 \\
(0.08 \%)\end{array}$ & 0.0551 & 0.1232 & -0.1028 & $\begin{array}{c}1.0487 \\
(-0.49 \%)\end{array}$ \\
\hline 40 & $\begin{array}{c}0.069785 \\
(0.08 \%)\end{array}$ & 0.0539 & 0.1255 & -0.1004 & $\begin{array}{c}1.0487 \\
(-0.49 \%)\end{array}$ \\
\hline 45 & $\begin{array}{c}0.096409 \\
(0.05 \%)\end{array}$ & -0.0198 & 0.2684 & 0.0184 & $\begin{array}{c}1.0532 \\
(0.02 \%)\end{array}$ \\
\hline 50 & $\begin{array}{c}0.096888 \\
(0.06 \%)\end{array}$ & -0.0210 & 0.2710 & 0.0208 & $\begin{array}{c}1.0532 \\
(0.02 \%)\end{array}$ \\
\hline 55 & $\begin{array}{c}0.098174 \\
(0.06 \%)\end{array}$ & -0.0242 & 0.2782 & 0.0275 & $\begin{array}{l}1.0531 \\
(0.01 \%)\end{array}$ \\
\hline
\end{tabular}

Table 6. The control performance of three Cases for IEEE 14-bus System

\begin{tabular}{cccc}
\hline & Case 1 & Case 2 & Case 3 \\
\hline$N_{\mathrm{C}}$ & 14 & $5(-64.29 \%)$ & $4(-71.43 \%)$ \\
$N_{\mathrm{K}}$ & 16 & $19(18.75 \%)$ & $31(93.75 \%)$ \\
$D\left(Q_{\mathrm{G} 1}\right)(\mathrm{pu})$ & 0.0398 & $0.0420(5.34 \%)$ & $0.0423(6.11 \%)$ \\
$D\left(Q_{\mathrm{G} 2}\right)(\mathrm{pu})$ & 0.0571 & $0.0845(47.96 \%)$ & $0.0867(51.93 \%)$ \\
$D\left(Q_{\mathrm{G} 3}\right)(\mathrm{pu})$ & 0.0406 & $0.0707(74.16 \%)$ & $0.0761(87.59 \%)$ \\
$\bar{P}_{\text {loss }}(\mathrm{pu})$ & 0.089950 & $0.089997(0.0526 \%)$ & $0.090038(0.0976 \%)$ \\
$\bar{V}(\mathrm{pu})$ & 1.0532 & $1.0522(-0.09 \%)$ & $1.0518(-0.13 \%)$ \\
\hline
\end{tabular}

demand is utilized, however, it brings in negative impacts, such as the increased $\bar{P}_{\text {loss }}$, almost double $N_{\mathrm{K}}$ and decreased $\bar{V}$. For $\bar{P}_{\text {loss }}$ and $\bar{V}$, Case 2 is very close to Case 1 and better than Case 3, which means a suitable voltage profile can be achieved by the proposed method.

\subsection{IEEE 118-bus system}

\section{Case 1}

Similar to IEEE 14-bus system, the optimization result is obtained for IEEE 118-bus system without considering the MADSON constraints.

As can be seen in Fig. 5, compensators are intended to inject relatively more banks at the first and last $15 \mathrm{~min}$ intervals, whereas relatively few at the middle $30 \mathrm{~min}$ intervals. This feature is consistent with pattern of load evolution. It is apparent that compensator 1 is maintained at the minimum value during the hour and other compensators are scheduled differently. On the other hand, transformer tap ratios are relatively stable during the hour compared with compensators. In other words, the voltage profile of this test system can be regulated appropriately by the compensators and generators. $P_{\text {loss }}$ and $\bar{V}_{\mathrm{L}}$ for each interval are presented in Table 7. Due to the limitation, $Q_{\mathrm{G}}$ is not shown for this test system.
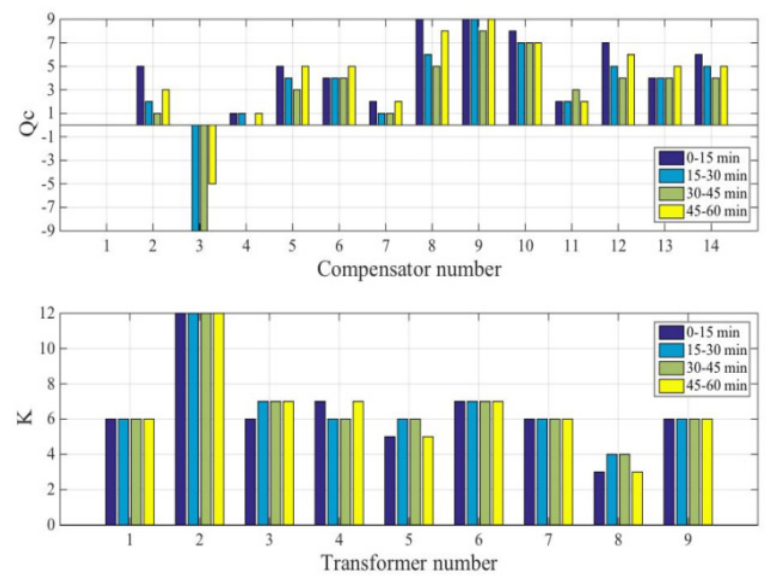

Fig. 5. The schedule of discrete control devices in Case 1

Table 7. Optimization result of Case 1

\begin{tabular}{ccc}
\hline Time $(\min )$ & $P_{\text {loss }}$ & $\bar{V}_{\mathrm{L}}$ \\
\hline 0 & 1.317759 & 1.0476 \\
5 & 1.309949 & 1.0477 \\
10 & 1.286937 & 1.0478 \\
15 & 0.875555 & 1.0500 \\
20 & 0.877279 & 1.0500 \\
25 & 0.871757 & 1.0500 \\
30 & 0.689953 & 1.0510 \\
35 & 0.695537 & 1.0510 \\
40 & 0.701144 & 1.0509 \\
45 & 1.084717 & 1.0488 \\
50 & 1.091463 & 1.0488 \\
55 & 1.110133 & 1.0487 \\
\hline
\end{tabular}




\section{Case 2}

In this case, the uncertainty is modeled using TPEM and the optimization result is presented in Fig. 6 and Table 8 .

From Fig. 6, the schedule of compensator 1 is as same as Case 1, which indicates the appropriate schedule for compensator 1 is also achieved by the proposed method under uncertainty effects. In addition, the schedule of transformer tap ratios is still stable under the fixed schedule of compensators. In Table $8, P_{\text {loss }}$ and $\bar{V}_{\mathrm{L}}$ come much close to Case 1 in most intervals, which means a suitable voltage profile is obtained due to the proposed method.

\section{Case 3}

The schedule of compensators is obtained based on the mean value of the one-hour load demand. It is observed from Fig. 7 that the schedule of transformer tap ratios is almost the same with Case 2 and Case 1. However, the schedule of compensators differs largely from other cases. In other words, in this test system, various compensator schedules can exist with little influence on the schedule of transformer tap ratios. Therefore, the coordination between compensators and generators would be the key issue for the desired voltage profile. Table 9 details $P_{\text {loss }}$ and $\bar{V}_{\mathrm{L}}$ in each interval.

Table 10 compared optimization results for the three cases. In Case $2, N_{\mathrm{C}}$ is reduced significantly due to the consideration of uncertainty and CACs. $N_{\mathrm{K}}$ and the total
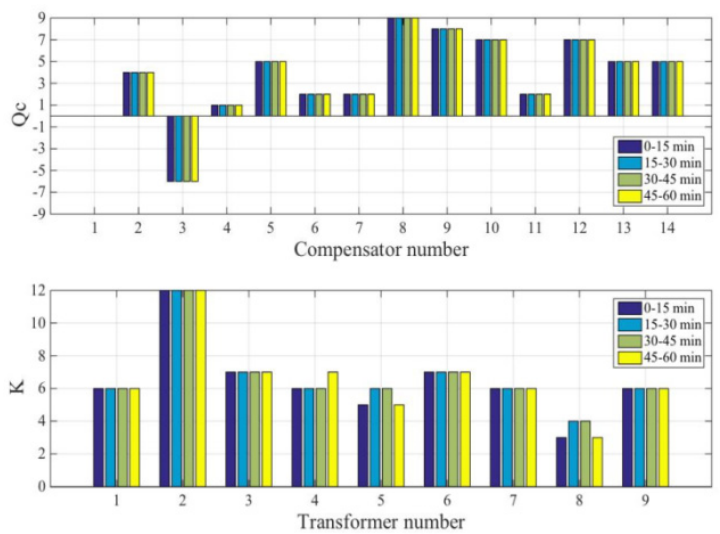

Fig. 6. The schedule of discrete control devices in Case 2

Table 8. Optimization result of Case 2

\begin{tabular}{ccc}
\hline Time $(\min )$ & $P_{\text {loss }}$ & $\bar{V}_{\mathrm{L}}$ \\
\hline 0 & $1.318112(0.03 \%)$ & $1.0475(-0.01 \%)$ \\
5 & $1.310284(0.03 \%)$ & $1.0476(-0.01 \%)$ \\
10 & $1.287222(0.02 \%)$ & $1.0477(-0.01 \%)$ \\
15 & $0.875588(0.00 \%)$ & $1.0501(0.01 \%)$ \\
20 & $0.877310(0.00 \%)$ & $1.0501(0.01 \%)$ \\
25 & $0.871793(0.00 \%)$ & $1.0501(0.01 \%)$ \\
30 & $0.690203(0.04 \%)$ & $1.0514(0.03 \%)$ \\
35 & $0.695769(0.03 \%)$ & $1.0513(0.03 \%)$ \\
40 & $0.701360(0.03 \%)$ & $1.0513(0.03 \%)$ \\
45 & $1.084758(0.00 \%)$ & $1.0488(0.00 \%)$ \\
50 & $1.091506(0.00 \%)$ & $1.0488(0.00 \%)$ \\
55 & $1.110180(0.00 \%)$ & $1.0487(0.00 \%)$ \\
\hline
\end{tabular}

operating times of compensator and OLTC are also reduced. In other words, frequent and excessive switching operations of discrete control devices can be avoided effectively. The increased mean value of standard deviation for all generator reactive power outputs $\bar{D}\left(Q_{\mathrm{G}}\right)$ clearly shows that the advantage of regulating characteristic of generators is taken by the proposed method.

Compare with Case 1. $\bar{P}_{\text {loss }}$ in Case 2 and 3 , is bound to increase due to the limited operating times of discrete control devices. However, in Case 2, the acceptable increase of $\bar{P}_{\text {loss }}$ indicates that the proposed method can achieve a similar voltage profile with Case 1 . Also, $\bar{V}$ is well regulated within the very small difference.

The proposed method utilized in Case 2 for the two test systems is performed on a PC with Inter(R) Core(TM) i7$47904.0 \mathrm{GHz}$ CPU. In this paper, GA is parallelized in Master-slave model to improve the computation perfor-
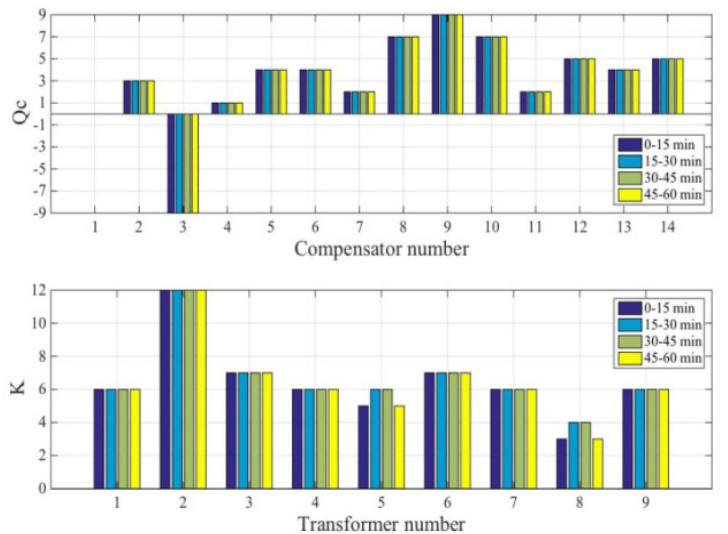

Fig. 7. The schedule of discrete control devices in Case 3

Table 9. Optimization result of Case 3

\begin{tabular}{ccc}
\hline Time $(\min )$ & $P_{\text {loss }}$ & $\bar{V}_{\mathrm{L}}$ \\
\hline 0 & $1.318789(0.08 \%)$ & $1.0475(-0.02 \%)$ \\
5 & $1.310933(0.08 \%)$ & $1.0475(-0.02 \%)$ \\
10 & $1.287793(0.07 \%)$ & $1.0476(-0.01 \%)$ \\
15 & $0.875552(0.00 \%)$ & $1.0500(0.00 \%)$ \\
20 & $0.877276(0.00 \%)$ & $1.0500(0.00 \%)$ \\
25 & $0.871755(0.00 \%)$ & $1.0500(0.00 \%)$ \\
30 & $0.689956(0.00 \%)$ & $1.0511(0.01 \%)$ \\
35 & $0.695532(0.00 \%)$ & $1.0511(0.02 \%)$ \\
40 & $0.701132(0.00 \%)$ & $1.0511(0.02 \%)$ \\
45 & $1.084883(0.02 \%)$ & $1.0488(-0.01 \%)$ \\
50 & $1.091639(0.02 \%)$ & $1.0487(-0.01 \%)$ \\
55 & $1.110339(0.02 \%)$ & $1.0486(-0.01 \%)$ \\
\hline
\end{tabular}

Table 10. The control performance of three cases for IEEE 118-bus system

\begin{tabular}{cccc}
\hline & Case 1 & Case 2 & Case 3 \\
\hline$N_{\mathrm{C}}$ & 49 & $13(-73.50 \%)$ & $18(-63.27 \%)$ \\
$N_{\mathrm{K}}$ & 7 & $5(-28.57 \%)$ & $4(-42.86 \%)$ \\
$\bar{D}\left(Q_{\mathrm{G}}\right)(\mathrm{pu})$ & 0.0398 & $0.0420(5.34 \%)$ & $0.0423(6.11 \%)$ \\
$\bar{P}_{\text {loss }}(\mathrm{pu})$ & 0.99268196 & 0.992840394 & 0.99296498 \\
$\bar{V}(\mathrm{pu})$ & 1.0494 & $(0.016 \%)$ & $(0.028 \%)$ \\
\hline
\end{tabular}


mance with 8 processors. The average computing time for IEEE 14-bus and IEEE 118-bus system are 93s and 413s, respectively, which has been reduced by $77.5 \%$ and $75.8 \%$ compared with the serial GA. Note that the calculation speed could be further sped up with more processors utilized in parallel GA. Therefore, the proposed method could satisfy the requirement for application.

\section{Conclusions}

This paper presents a two-stage stochastic programming model for optimal reactive power dispatch with high penetration level of wind generation. The potential impacts of uncertainty of wind speed and load on voltage control performance are regarded by converting a DORPD model into a two-stage stochastic programming problem while considering the costs of adjusting the compensators.

The stochastic behavior of wind speed and load is simulated using TPEM. To solve this large-scale mixedinteger nonlinear stochastic programming, a hybrid GAIPM is developed, in which GA deals with the discrete variables and IPM achieves the fast convergence. Additionally, parallel GA and a quadratic DORPD model are utilized to reduce the computational burden. The proposed method is implemented and analyzed on the IEEE 14-bus and IEEE 118-bus system. Case studies show that excessive operations of compensators are avoided effectively due to the consideration the uncertainty and CACs. The total operating times of discrete control devices is reduced as well. The similar voltage profile of system is achieved with the acceptable expense of a small increase in real power loss.

\section{Acknowledgements}

This work was supported by the National Natural Science Foundation of China (51677012), and the Fundamental and Frontier Research Foundation of Chongqing (cstc2013 jcyjA90001).

\section{References}

[1] Y. Hsu and H. Kuo, "Dispatch of capacitors on distribution system using dynamic programming," Generation, Transmission and Distribution, IEE Proceedings $C_{2}$ vol. 140, no. 6, pp. 433-438, Nov. 1993.

[2] Y. Hsu and C. Yang, "A hybrid artificial neural network-dynamic programming approach for feeder capacitor scheduling," IEEE Trans. Power Systems, vol. 9, no. 2, pp. 1069-1075, May 1994.

[3] F. Lu and Y. Hsu, "Fuzzy dynamic programming approach to reactive power voltage control in a distribution substation," IEEE Trans. Power Systems, vol. 12, no. 2, pp. 681-688, May 1997.

[4] Y. Hsu and F. Lu, "A combined artificial neural network-fuzzy dynamic programming approach to reactive power voltage control in a distribution substation," IEEE Trans. Power Systems, vol. 13, no. 4, pp. 1265-1271, Nov. 1998.

[5] M. B. Liu, C. A. Canizares and W. Huang, "Reactive Power and Voltage Control in Distribution Systems With Limited Switching Operations," IEEE Trans. Power Systems, vol. 24, no. 2, pp. 889-899, May 2009.

[6] T. Malakar and S. K. Goswami, "Active and reactive dispatch with minimum control movements," International Journal of Electrical Power \& Energy Systems, vol. 44, no. 1, pp. 78-87, Jan. 2013.

[7] D. Youman, Z. Boming and T. Tian, "A Fictitious Load Algorithm and It's Applications to Distribution Network Dynamic Optimizations," Proceedings of the CSEE, vol. 16, no. 4, pp. 241-244, Jul. 1996.

[8] Z. Hu, X. Wang, H. Chen, and G. A. Taylor, "Volt/ VAr control in distribution systems using a timeinterval based approach," Generation Transmission and Distribution, IEE Proceedings-, vol. 150, no. 5, pp. 548-554, Sept. 2003.

[9] H. Luo, M. Chen and T. Cheng, "Adaptive TimeIntervalled Reactive Power Optimization for Distribution Network Containing Wind Power Generation," Power System Technology, vol. 38, no. 8, pp. 22072212, Aug. 2014.

[10] Y. J. Zhang and Z. Ren, "Optimal Reactive Power Dispatch Considering Costs of Adjusting the Control Devices," IEEE Trans. Power Systems, vol. 20, no. 3, pp. 1349-1356, Aug. 2005.

[11] Z. Hu, X. Wang and G. Taylor, "Stochastic optimal reactive power dispatch: Formulation and solution method," International Journal of Electrical Power \& Energy Systems, vol. 32, no. 6, pp. 615-621, Jul. 2010.

[12] S. M. Mohseni-Bonab, A. Rabiee, B. MohammadiIvatloo, S. Jalilzadeh, and S. Nojavan, "A two-point estimate method for uncertainty modeling in multiobjective optimal reactive power dispatch problem," International Journal of Electrical Power \& Energy Systems, vol. 75, pp. 194-204, Feb. 2016.

[13] R. Liang, J. Wang, Y. Chen, and W. Tseng, "An enhanced firefly algorithm to multi-objective optimal active/reactive power dispatch with uncertainties consideration," International Journal of Electrical Power \& Energy Systems, vol. 64, pp. 1088-1097, Jan. 2015.

[14] A. T. Saric, F. H. Murphy, A. L. Soyster, and A. M. Stankovic, "Two-Stage Stochastic Programming Model for Market Clearing With Contingencies," IEEE Trans. Power Systems, vol. 24, no. 3, pp. 1266-1278, Aug. 2009.

[15] P. Xiong and P. Jirutitijaroen, "Two-stage adjustable robust optimisation for unit commitment under uncertainty," IET Generation, Transmission \& Distri- 
bution, vol. 8, no. 3, pp. 573-582, Mar. 2013.

[16] E. M. Constantinescu, V. M. Zavala, M. Rocklin, S. Lee, and M. Anitescu, "A Computational Framework for Uncertainty Quantification and Stochastic Optimization in Unit Commitment with Wind Power Generation," IEEE Trans. Power Systems, vol. 26, no. 1, pp. 431-441, Feb. 2011.

[17] J. R. Soto, C. Domellas and D. M. Falcao, "Optimal reactive power dispatch using a hybrid formulation: genetic algorithms and interior point," in Power Tech Proceedings, 2001 IEEE Porto, vol. 3, pp. 5, Sept. 2001.

[18] Birge, J. R. and Louveaux, F, Introduction to Stochastic Programming: Springer Science \& Business Media, 2011.

[19] Y. Li, W. Li and W. Yan, "Probabilistic Optimal Power Flow Considering Correlations of Wind Speeds Following Different Distributions," IEEE Trans. Power Systems, vol. 29, no. 4, pp. 1847-1854, Jul. 2014.

[20] Hong H P. "An efficient point estimate method for probabilistic analysis," Reliability Engineering \& System Safety, vol. 59, no. 3, pp. 261-267, Jul. 1998.

[21] Cant-Paz E. "A survey of parallel genetic algorithms," Calculateurs Paralleles, Reseaux Et Systems Repartis, vol. 10, pp. 141-171, May 1998.

[22] W. Yan, J. Yu, D. C. Yu, and K. Bhattarai, "A new optimal reactive power flow model in rectangular form and its solution by predictor corrector primal dual interior point method," IEEE Trans. Power Systems, vol. 21, no. 1, pp. 61-67, Feb. 2006.

[23] MATPOWER [Online]. Available: http://www.pserc.cornell.edu/matpower,

[24] H. Sun, Q. Guo, B. Zhang, W. Wu, and B. Wang, "An Adaptive Zone-Division-Based Automatic Voltage Control System With Applications in China," IEEE Trans. Power Systems, vol. 28, no. 2, pp. 1816-1828, May 2013

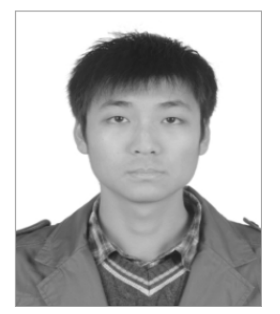

Wei Cui $\mathrm{He}$ is currently working towards the Ph.D. degree at the College of Electrical Engineering, Chongqing University, Chongqing, China. His research interests include voltage optimization and control, and distributed generation.

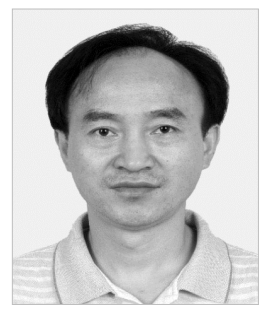

Wei Yan He received the Ph.D. degree in electrical engineering from Chongqing University, Chongqing, China, in 1999. Currently, he is a Professor in the Electrical Engineering College at Chongqing University. His research interests include optimal operation and control in power systems.

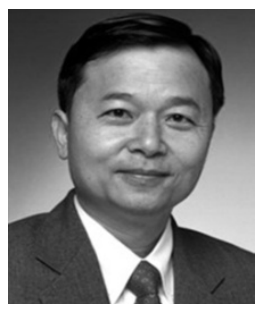

Wei-Jen Lee He received the B.S. and M.S. degrees from National Taiwan University, Taipei, Taiwan, R.O.C., and the Ph.D. degree from the University of Texas, Arlington, in 1978, 1980, and 1985, respectively, all in Electrical Engineering. In 1985, he joined the University of Texas, Arlington, where he is currently a professor of the Electrical Engineering Department and the director of the Energy Systems Research Center. He has been involved in research on power flow, transient and dynamic stability, voltage stability, short circuits, relay coordination, power quality analysis, renewable energy, and deregulation for utility companies. Prof. Lee is a Fellow of IEEE and registered Professional Engineer in the State of Texas.

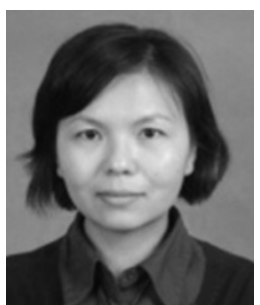

Xia Zhao She received the Ph.D. degree in electrical engineering from Chongqing University, Chongqing, China, in 2009. Currently, she is an Associate Professor with the Electrical Engineering College at Chongqing University. Her research interests include power system risk analysis and distributed generation modeling and analysis.

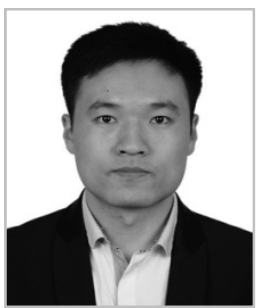

Zhouyang Ren He received the Ph.D. degree in electrical engineering from Chongqing University, Chongqing, China, in June 2014. Currently, he is a Lecturer in the Electrical Engineering College at Chongqing University. He is also a researcher in the Power and Energy Reliability Research Center of Chongqing University. His research interests include probabilistic applications to power systems, power system reliability assessment, and power system data analysis.

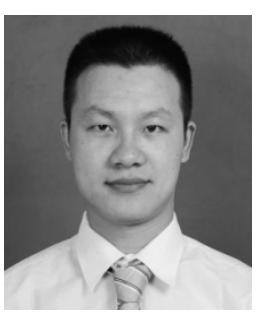

Cong Wang He is currently working towards the Ph.D. degree at the College of Electrical Engineering, Chongqing University, Chongqing, China. His research interests include intelligent control, distribution power network optimal operation and planning. 